\title{
The Precursor to an Industrial Software Metrics Program
}

\author{
S.T. Parkinson, S. Counsell, M. Norman, R. M. Hierons and M. Lycett, \\ School of Information Systems, Computing and Mathematics, \\ Brunel University, Uxbridge, Middlesex. UB8 $3 P H$. \\ Email: steve.counsell@brunel.ac.uk
}

\begin{abstract}
A common reason for why software metric programs 'fail' is through lack of participant support and commitment. In this paper, we describe the results of a study which examined the knowledge that subjects had and the opinions they had formed of previous metrics initiatives in the same organization. The research was undertaken by one of the authors as a precursor to a planned metrics initiative in the same large, UK-based company. The study attempted to understand the likely issues that would have to be addressed by that planned metrics program. A key theme to emerge from the analysis was the importance of all participants being aware of the program objectives, and the purpose and use of the data being collected. As part of the analysis, the study also draws on the role that 'timely' involvement plays within a metrics program and how that can influence its associated practicalities.
\end{abstract}

Keywords. Software Metrics, Industry Practice.

\section{Introduction}

One of the main reasons why metrics programs often fail to deliver the benefits they promise is lack of enthusiasm and support for the scheme amongst the employees participating in the program $[13,14]$. At best, the record of metrics programs in industry is poor [17]. One way of understanding these critical issues is to survey the opinions of relevant staff members on their views of prior programs to inform future studies. Of particular interest in the study described herein are the views on previous programs by developers, project managers and IT managers. Decisions are often made and aims and objectives are often set by the latter without filtering through to the 'data gatherers' (i.e., the developers). Paradoxically, it is in the hands of exactly these data gatherers that the success or failure of the same program can rest. In this paper, we describe an approach adopted by an IT group of a large, UK-based company in which employees were asked about their knowledge of previous metrics programs. One of the authors worked in the company where the group was based and undertook the investigation through the use of questionnaires and interviews [20]. The purpose of the study was to assess the likely problems an impending metrics program would face by involving the developers more heavily in the goal-setting process. Not surprisingly, the investigation revealed a lack of appreciation and knowledge by the developers of previous program's objectives and/or the reasons why the relevant data was being collected/how it was being used. Quotes from staff suggest that developers would embrace an initiative if only they were given the proper information.

\section{Related work}

The research described in this paper addresses some of the issues that arise when considering an industrial metrics program. Anecdotal evidence on the success of metrics programs suggests that $75 \%-80 \%$ or more of metrics programs fail to deliver their objectives [7]. The importance of support amongst management for a metrics program is also stressed in [21]. The responsibility on the part of the developer in terms of generating metrics data and working towards the goals of the program is also seen as an important feature [2, 3, 8, 12, 14]. The notion that practitioners should be aware of what metrics are being collected and should be actively encouraged to participate is described in [14]. The research described in this paper is also linked to Software Process Improvement (SPI) [15]. Research in SPI suggests that the experience levels of a workforce are a major contributor to the success of process initiatives. While this may provide an indication of a similar effect on metrics program implementations, there is some evidence to suggest that experience places extra demands in such circumstances and a range of additional activities are necessary to 
ensure acceptance by staff [18]. Moreover, traditional research methods do not appear to have advanced theory into practical benefit for the IS industry [9, 10, 11, 19]. Varieties of implementation methods have been proposed, but the success rate of metrics programs still remains poor. If research into metrics programs is to add any value to the extensive literature on the subject, practical relevance of the metrics to those involved should be a key driver. Although the study does not specifically represent action research in its strictest sense $[1,4,5,6]$, the underlying aim of improving practices and strategies mean there are some common elements.

\section{The study environment}

The Computing Solutions (CS) group is an established IT department of a large, UK-based company and provides IT development support to its clients within the UK. The managers within the company aim to provide a responsive IT service which maximizes the return on business investment in IT in the short term. Although company-wide attempts were being made to adopt a formal CMM-based SPI program $[15,16]$, it was generally felt that a more flexible metrics-based improvement approach was in keeping with the shorter term goals of CS. Like many large organizations of its type within the UK, the company has recently been involved in acquisitions and mergers. This has placed a significant emphasis within CS on integrating large complex legacy systems, development processes and software development departments. CS employs an experienced workforce and has high staff retention rates, but does not currently deploy a formal structured metrics program (it has attempted to implement such a program in the past). The study described involved the use of a 'pilot' questionnaire, the results of which would inform a 'live' questionnaire distributed to a larger sample of staff at CS.

\subsection{The pilot questionnaire}

In order to increase the likelihood that the live questionnaire would extract the desired information from the staff at CS, a pilot questionnaire using a small sample of support staff from the same department was distributed. The questionnaire was designed to establish the views of staff at CS and determine whether the inclusion of practitioners more actively in goalsetting would be of benefit. Each question in the questionnaire required the respondent to select one or more responses from a list. The questions were interspersed with a number of free-format areas which provided respondents with the opportunity to present their views on their experiences of metrics programs. As an organization with many experienced staff, free format areas provided an effective means of eliciting the views of individuals at CS. The pilot questionnaire was distributed with the following five objectives:

1. To ensure that the context of the work was clearly understood.

2. To ensure clarity in what was being asked of the respondent in each question.

3. To ensure that the respondents' understanding of each question matched that of the researcher.

4. To ensure that time for questionnaire completion was reasonable.

5. To ensure that the style of questionnaire was deemed appropriate for all intended recipients in CS.

Six participants were selected to pilot the questionnaire and were drawn from a single team at CS. This represents approximately $15 \%$ of the number of the participants who responded to the subsequent live questionnaire (see Section 4). In terms of composition, the pilot team was a support function for the whole of CS and had broad ranging responsibilities. The pilot participants also had a number of specific characteristics that made them ideal for the pilot: a) they had an experience profile similar to the majority of staff in CS, b) they had direct experience of working in a variety of areas of CS in their day-to-day activities and could thus provide an insight into how the staff may react to a later 'live' questionnaire and c) they had a broad range of IT skills.

\section{The 'live' questionnaire}

The live questionnaire was issued to all the permanent staff at CS, a total of 72. The 72 potential respondents comprised 5 decision makers and 67 practitioners. The responses were broken down into three roles, namely, IT manager, project manager and developer. The first section of the questionnaire comprised three closed questions asking the subjects for basic 
information. The responses to the three questions in Section 1 facilitated the categorization of respondents into practitioners (the project managers and developers) and decision makers (IT managers). This section also helped determine the respondent's level of IT experience. It was felt that the more experienced members of staff would provide a richer source of information regarding previous metrics programs at $\mathrm{CS}$; when combined, the answers from this section of the questionnaire would identify the most effective project(s), system or team to approach to host the eventual metrics program. Section 2 of the questionnaire asked the respondent to focus upon their experience of previous metrics program implementations. The questionnaire aimed to identify the implementation approaches adopted previously. Asking the respondents to indicate whether, at the time of the program implementation, they were aware of the objectives of the metrics program helped to identify the approaches previously adopted within CS. Question 5 asked about the range of IT measures that participants were familiar with in previous metrics programs. It was felt that reusing well-understood measures wherever possible would maximize the simplicity and effectiveness of the pilot metrics program. Question 7 was linked to question 5 and related to the communication surrounding the metrics program. To ensure that the respondents did not confuse the program objectives and how the measures were actually used, these questions were separated. The final question in this section asked the respondents to indicate why they felt failure of previous programs had become part of the IT culture at CS.

\subsection{Questionnaire results}

Overall, 39 of the 72 participants responded (i.e. $54 \%$ of those asked). Of these 39,4 were IT managers, 7 were project managers and 28 were developers. Due to the confidentiality promised to the recipients of the questionnaire, no followup investigation was possible to determine the specific reasons for the low response rate. However, one factor worth noting is the timing of the questionnaire. CS has a series of major software releases throughout the year and the period of the questionnaire coincided with one such software release. Those involved in the release had a significantly lower response rate (29\%) than those involved in development (65\%) at such a crucial stage in the delivery cycle. This information was volunteered in an email from the IT Manager involved in the release, where it was indicated that it was unlikely that anyone in their area of responsibility would have had sufficient time to respond. The length of service of the respondents may also be factor when determining the wider effectiveness of the approach; 38 of the 39 respondents $(97.4 \%)$ had worked for the organization for ten or more years. The knowledge that this group had unquestionably acquired on previous metrics programs would prove invaluable for the credibility of the study.

\subsubsection{Knowledge of program objectives}

A key question of the research was whether a common understanding of the metrics program objectives and the use of the gathered data would have a positive effect on the success of a metrics program. Fig. 1 shows that almost a third (31\%) of the non-project management practitioners were not aware of the reasons for collecting the data in the metrics programs they had previously been involved with. As you might expect from a traditional 'top-down' implementation with little or no information filtering down to lower levels, all decision makers and project managers were aware of the objectives of the previous programs.

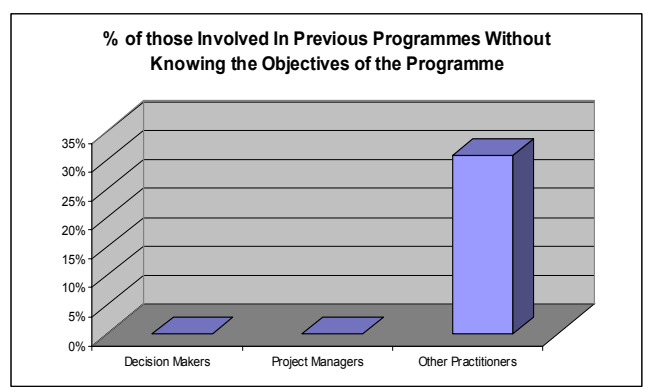

Figure 1. Knowledge of previous objectives

\subsubsection{Knowledge about data usage}

To investigate the level of communication of the different approaches, the questionnaire sought views on whether the participants knew how previous programs used the gathered data. Fig. 2 shows the breakdown of the response to this question. Although $100 \%$ of decision makers knew how the data was used, only $29 \%$ of project managers and $24 \%$ of developers had a similar understanding. Again this highlights the nature of a top-down approach to metrics 
programs and, although the objectives of the program were communicated at the project management level, information on the data usage rarely made it beyond this group (i.e. the nonmanagement group of practitioners was largely ignored). It is unclear whether this was a conscious decision of the metrics program; if it was, then it would seem to contradict good practice in metrics programs. On the other hand, it may simply be that the relevant information was expected to 'trickle down' to the nonmanagement group of practitioners without any formal, information distribution framework. In such a case, the shortcoming may lie with the absence of such a framework, rather than any deliberate intention on the part of the decisionmakers to withhold information.

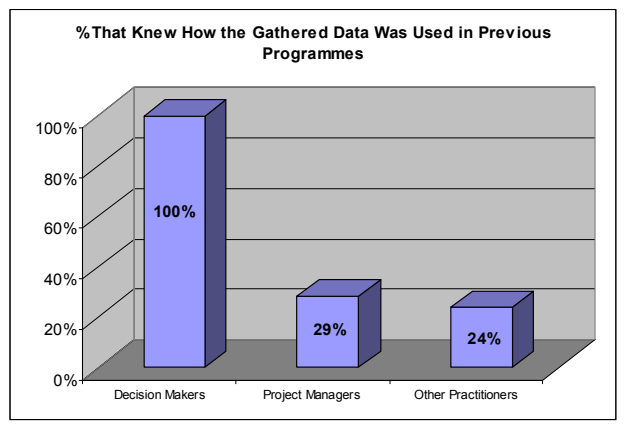

\section{Figure 2. Knowledge of previous programs}

Although poor communication may account for the lack of awareness of how the data was used, the very low percentages suggest that there is more to the results than simply poor communication. Finally, in common with the results shown in Fig. 1, knowing why the data is being collected is as important as knowing the objectives of the overall program; lack of knowledge of both can be equally detrimental to a metrics program.

\subsubsection{Beneficiaries of a metrics program}

Another important objective of the study was to establish views of the questionnaire subjects on who they thought the beneficiaries of a metrics program might be. Fig. 3 shows the views of just the developers on this issue. Developers view project managers and IT managers to be the main beneficiaries of a metrics program $(82 \%$ of respondents thought that project managers were the chief beneficiaries).

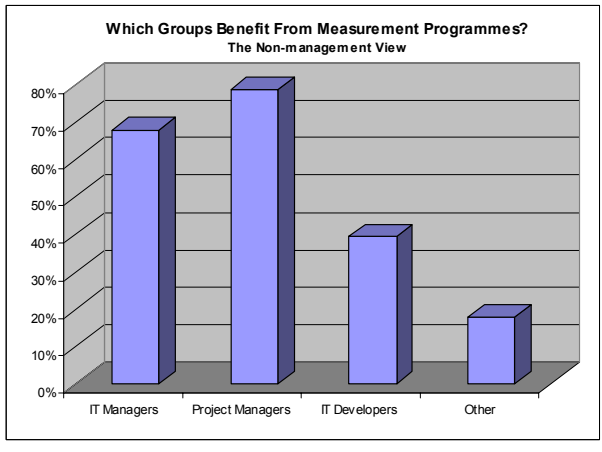

Figure 3. Developer view of beneficiaries

Only $39 \%$ of developers thought that they themselves were the beneficiaries of a metrics program. A strong sense of the disaffection felt by developers is thus evident from Fig. 3 and perhaps this is not surprising based on the results from Figs. 1 and 2. If the participants of a metrics program are not aware of either the objectives of the program or why the data is being collected (and how that data is being used), it is unlikely that they will view that program as being for their benefit. Fig. 4 shows the perspective from the management view (IT managers and project managers) and shows that $100 \%$ in this group thought that it was they themselves who benefited from a metrics program; only slightly fewer IT managers and project managers thought that developers also benefited from the same program.

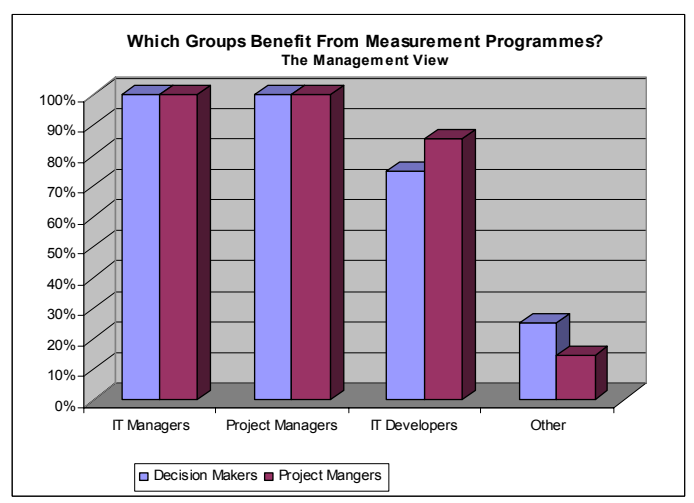

Figure 4. Management view of beneficiaries

The result from Fig. 4 is also not surprising considering the results from Figs. 1, 2 and 3. On reflection, it is however unlikely that the high level of management expectations in regard to the benefit of a metrics program to developers is going to be met when more than $2 / 3$ of developers (Fig. 3) do not consider this an outcome and even less (Fig. 2) have in the past been aware of (and presumably therefore not 
involved with) the use made of the metrics. One could view the attitude of the IT managers and project managers as, at best, mistaken and, at worst, short-sighted. We suggest that the quality and value of data generated by a metrics program can be as influenced by the active involvement and enthusiasm of the data gatherers as by other issues [13].

\subsubsection{Program objectives and importance}

Fig. 5 shows two groups of respondents; the first is the set of practitioners who had indicated that they were aware of the objectives of prior metrics programs and who had responded 'Critical' or 'Important' when asked how useful a metrics program would be in CS (the rankings were: 'An unnecessary overhead', 'Of some benefit', 'Useful', 'Important' and 'Critical'). The second group is the set of practitioners who indicated that they were not aware of the objectives of prior metrics programs and who had also responded either 'Critical' or 'Important'. We note that decision makers were excluded since they were all aware of the objectives of the previous programs. From Fig. 5, it can be seen that $32 \%$ of the group who were aware of previous metrics program objectives thought a metrics program would be useful; only $13 \%$ of the group who were not aware of previous metrics program objectives thought a metrics program useful. The clear message from Fig. 5 is that if practitioners are made aware of the objectives of the program, then they are likely to view the program as important as a whole.

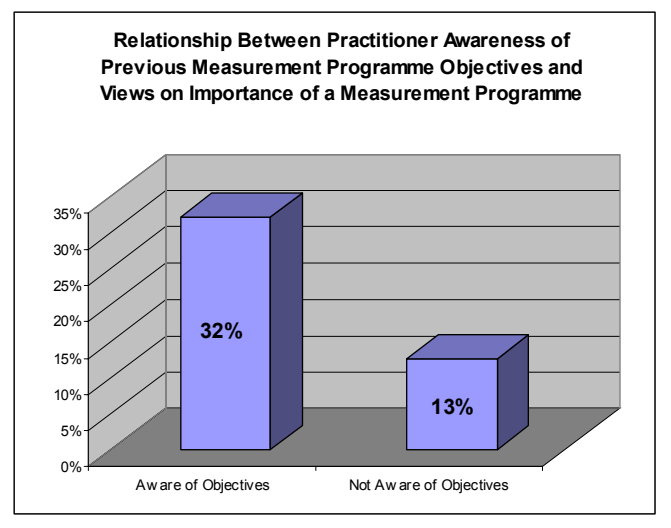

Figure 5. Awareness of objectives

We could suggest that the objectives of the program heavily inform the type of data gathered for the program; if practitioners are made aware of the program objectives, the quality of collected data may improve as a result and the more effective the program as a whole will be.

\subsection{The importance of timely involvement}

Following the analysis as part of the questionnaire described, a pilot metrics program was introduced requiring the selection of a new pilot team. A presentation was given to members of this team explaining the results of the questionnaire and precise details about the procedures for implementing the pilot program. For example, the team was given information on 'Getting Started', 'Goal Setting' and the 'Next Steps'. During the early stages of the pilot program, the investigator undertook a series of semi-structured interviews lasting between five and twenty minutes the aim of which was to establish the views of the pilot team on the setting of program goals and their overall impression of the program so far. A number of key themes emerged from these interviews which are missing from many metrics programs and illuminate the reasons why other metrics programs fail. Developers appreciated firstly, being involved at early stages of the program and secondly, given the opportunity to contribute. Some quotes to support this are: "Developers will buy in if they are involved early". (Interviewee 1) and "(previous programs made) no attempt to get people on board and explain it (the program objectives)". (Interviewee 2) and "Give people a chance to input". (Interviewee 3 )

"..........engaging the practitioner will produce much better results.......(if you) get them involved at the beginning they can easily identify areas of improvement". (Interviewee 4) and "(previously) no-one ever asked us our opinions in terms of what it (the metrics program) was trying to achieve........it was difficult to see where it was going..........it's a positive move asking practitioners to contribute" (Interviewee 4). There was also evidence that the developers themselves would respond more readily if they were involved more and a more empathetic view of their position taken: "....(practitioners need)....time to gather stats.....(otherwise) it's the kind of thing that could get pushed to one side (due to priorities)" (Interviewee 5).

It is clear from these quotes that developers were keen to contribute to any metrics program, but felt frustrated that, in the past, they had not 
been given much chance to. Such a conclusion would be consistent with the data in Figs. 1 to 5 . A large degree of the value of the study described in this paper can be attributed to 'opening up' a debate on the issues that seem to concern developers prior to the implementation of a metrics program. Good practice should enforce a preliminary study where the views of all concerned can be expressed and grievances aired. We also feel that adopting 'action research-like' studies has immense value for informing industry practice and bridging the gap between industry and academia.

\section{Conclusions}

In this paper, we described a study of the views of IT managers, project managers and developers in an IT group of a large UK-based company. A questionnaire about previous metrics programs was distributed and interviews undertaken with members of staff. The main theme to emerge from the analysis was the importance attached to developers being made fully aware of the program objectives, the reasons why the data was being collected and how the data was subsequently used. A striking feature of the analysis was the low percentage of project managers and developers with knowledge about how the metrics program data was actually going to be used. An over-arching conclusion from our study is the necessity for an equal stake in the development and implementation of a metrics program for all involved in the process.

\section{References}

[1] D. Avison, R. Baskerville, and M. Myers, Controlling action research projects. Inf. Tech. \& People, 14(1), pp. 28-45. 2001.

[2] N. Baddoo, and T. Hall. De-motivators for software process improvement: an analysis of practitioners' views. Journal of Systems and Software, 66(1), pp. 23-33, 2003.

[3] V. Basili and D. Rombach. The TAME project: towards improvement-oriented software environments. IEEE Trans. on Software Eng., 14(6):758-773. 1988.

[4] R. Baskerville and A. Wood-Harper. Diversity in Information Systems Action Research Methods. European Journal of Inf. Systems, 7(2):90-107, 1998.

[5] R. Baskerville. Investigating IS with action research. Comms AIS, 2(3es), pp. 4.
[6] R. Baskerville and J Pries-Heje. Grounded action research: a method for understanding IT in practice. Accounting, Management and Inf. Technologies, 9(1), pp. 1-23. 1999.

[7] C. Dekkers and P. McQuaid, The dangers of using software metrics to (mis)manage. IT Professional, 4(2), pp. 24-30. 2002.

[8] N. Fenton. Software measurement: a necessary scientific basis. IEEE Trans. on Soft. Engineering, 20(3), pp. 199-206. 1994.

[9] N. Fenton and M. Neil. Software metrics: successes, failures and new directions. Journal of Systems and Software, 47(2-3), pp. 149-157. 1999.

[10] R. Galliers, Choosing Information Systems Research Approaches. Information Systems Research. Blackwell Scientific Publications, pp. 144 -166. 1992.

[11] R. Glass. The relationship between theory and practice in software engineering. Comm. of the ACM, 39(11), pp. 11-13. 1996.

[12] D. Goldensen, A. Gopal and T. Mukhopadhyay. Determinants of success in software measurement programs: initial results, 1999, pp. 10-21. 1999.

[13] T. Hall and N. Fenton. Implementing Software Metrics - The Critical Success Factors. Soft. Qual. Jour., 3(1):195-208.

[14] T. Hall and N. Fenton. Implementing effective software metrics programs. IEEE Software, 14(2):55-65. 1997.

[15] J. Herbsleb and D. Goldensen. A systematic survey of CMM experience and results, Proceedings of Intl. Conf. on Soft. Eng., 1996, IEEE Comp. Soc. pp 323-330. 1996.

[16] J. Herbsleb, D. Zurbrow, D. Goldensen, W. Hayes and M. Paulk. Soft. quality and the Capability Maturity Model. Comms. of the ACM, 40(6):30-40. 1997.

[17] W. Hetzel. The sorry state of software practice measurement and evaluation. Jour. of Sys. and Soft., 31(2), pp. 171-179. 1995.

[18] E. McGuire. Factors affecting the quality of software project management: an empirical study based on the Capability Mat. Model, Soft. Qual Jour., 5(4):305-317, 1996.

[19] L. Osterweil, Strategic directions in software quality. ACM Comp..Surveys., 28(4):738-750, 1996.

[20] S. T. Parkinson, Implementing Software Measurement Adopting a Collaborative Approach, MPhil Thesis, Brunel Univ, 2006.

[21] S. Pfleeger. Lessons learned in building a corporate metrics program. IEEE Software, 10(3), pp. 67-74, 1993. 